\title{
Cosmological CP violation
}

\author{
Roman Tomaschitz \\ Physics Department, University of the Witwatersrand WITS 2050, Johannesburg, \\ South Africa and Department of Physics, Hiroshima University, \\ Higashi-Hiroshima 724, Japan
}

(Received 10 June 1993; accepted for publication 7 December 1993)

\begin{abstract}
Spinor fields are studied in infinite, topologically multiply connected RobertsonWalker cosmologies. Unitary spinor representations for the discrete covering groups of the spacelike slices are constructed. The spectral resolution of Dirac's equation is given in terms of horospherical elementary waves, on which the treatment of spin and energy is based in these cosmologies. The meaning of the energy and the particle-antiparticle concept is explained in the context of this varying cosmic background. Discrete symmetries, in particular inversions of the multiply connected spacelike slices, are studied. The violation of the unitarity of the parity operator, due to self-interference of $P$-reflected wave packets, is discussed. The violation of the $C P$ and $C P T$ invariance-already on the level of the free Dirac equation on this cosmological background-is pointed out.
\end{abstract}

\section{INTRODUCTION}

Cosmology - so far as the global structure of the universe is concerned-has always been somewhat at odds with the basic criterion of verifiability, that we rightly impose, since Galilei's time, onto a physical discipline.

This has lead to a very cautious attitude of many eminent physicists toward cosmological modeling. So, for example, the idea that the universe is infinite has been rejected on the grounds that we will not be able to look at infinity, and to verify what is happening there. ${ }^{1}$ Other quite attractive arguments, like the atomicity of matter, ${ }^{2}$ or Mach's principle, ${ }^{3}$ were put forward to plead for the closure of space. A noticeable exception is Ref. 4. I think it is also fair to say, that closed universes are handier for heuristic reasoning.

Although we are not able to look at infinity, there may be nevertheless the possibility to test infinite cosmological models, provided that local, microscopic phenomena are influenced by the global topological and metrical structure of the universe. It may be also appropriate to mention here that over the centuries most natural philosophers attached to the concept "Universe" the attribute "infinite," and some kind of nontrivial evolution toward a nontrivial end.

Consequently we should abandon the reasoning "we do not know the global structure, and therefore we assume the simplest possible." Instead we have to try different topological and metrical scenarios, and to find traces of them in the quantum fields, and then we have to see what makes the difference.

In practice this means that we should leave in our arguments the details of the global structure as long as possible unspecified, for example, the specific structure of the generators of the covering group of the spacelike slices, similarly as is usually done with the expansion factor in the topologically trivial models.

We must keep in mind that the ultimate question is not so much what is the global structure of the universe, but rather how does it evolve. In fact, our basic assumptions, ${ }^{7}$ that space is infinite, that it is multiply connected, and that it has constant negative curvature, match well in this respect. They make a dynamic evolution of the metric ${ }^{8,9}$ (by global deformations of the three-space manifold which do not change the curvature, but lead to nonisometric spacelike slices), and ultimately also transitions from one topology to another (there is some similarity with Wheeler's superspace ${ }^{3}$ ) possible. In closed universes with compact hy- 
perbolic manifolds as spacelike slices such deformations are not possible (Mostow rigidity theorem ${ }^{10}$ ), without introducing distortions of the curvature. (However, a closed multiply connected universe with Euclidean three-sections may well undergo global deformations. ${ }^{9}$ )

Even if we do not know the actual laws of the cosmic evolution yet, we can nevertheless make some preliminary conclusions. ${ }^{9}$ Open Robertson-Walker (RW) universes with multiply connected hyperbolic spacelike slices have a finite chaotic center, ${ }^{7}$ that in my opinion is the reason for the remarkable though apparently imperfect uniformity of the galactic background. Global metrical deformations lead to annihilation and production processes in wave fields, ${ }^{11}$ which are otherwise freely propagating in space. In particular such deformations can lead to particle production processes in neutrino fields, which cannot be generated by a mere variation of the expansion factor, cf. Example 2 in Sec. VI, where positive and negative frequencies stay perfectly well defined. ${ }^{12}$ The angular anisotropy in the temperature of the microwave background is likewise a natural consequence of a dynamic evolution by metrical deformations. ${ }^{8}$

In this article we report on another striking topological phenomenon: parity, the spacereflection symmetry, is violated on the most fundamental level, namely, in the free Dirac equation on a multiply connected cosmological background. There is substantial evidence that $C, P$, and $T$ are not generic symmetries on the microscopic level. Quantum field theory can cope easily with that, but to describe it one has to add deliberately symmetry breaking interaction terms to the Lagrangians. Concerning neutrinos, if we describe them by four-component spinors, we must exclude without $a$ priori reason half of the possible solutions of the Dirac equation. Two-component equations, cf. Refs. 13 and 14, constitute a possible adaptation to this situation, but do not really provide a reason why there are no right-handed neutrinos and left-handed antineutrinos. Why should left-handed neutrinos and right-handed antineutrinos be preferred to their counterparts, as long as there is no natural mechanism to break the space reflection or charge conjugation symmetry?

Now, the $T$ symmetry is violated because of the expansion of the space. That is actually not a surprising thing, and occurs in practice also in classical probabilistic systems. In order to render such classical systems time symmetric one would have to prepare initial and end value conditions with infinite precision. In fact, geodesic motion in this context is a good example for that. ${ }^{15}$

The violation of the space-inversion symmetry has now really no classical counterpart. It is an interference phenomenon, that stems from the fact that in a multiply connected space a $P$-reflected wave packet can overlap with itself. Finally, $C$ is still a good symmetry of the free Dirac equation, but the unitarity of $C P$ and noticeably $C P T$ is violated. (For the definition of these operations in hyperbolic space see Sec. V.) Clearly one is tempted to speculate if this can lead, if combined with particle annihilation-creation processes (which occur likewise in the free Dirac equation, cf. Example 3 in Sec. VI), to a dynamic generation of the baryon asymmetry in the universe. But it is outside the scope of this article to give quantitative evidence, and we will also not discuss how this topological symmetry breaking relates to current particle phenomenology. In particular we will not address the important question why $C P$ violation appears to be so weak that it has up to now only been confirmed in kaon systems. Our main objective is to show, that $P$ is not a natural symmetry on the microscopic level, as soon as one passes from the classical point-particle concept to wave equations in the context of a multiply connected cosmology.

The article is organized as follows. In Sec. II we study the continuous symmetries of the Dirac equation in hyperbolic space $H^{3}$. They are generated by the orientation preserving invariance group $\operatorname{SL}(2, \mathbb{C}) /\{ \pm$ id $\}$ of the hyperbolic metric. We construct the spinor representation of $\operatorname{SL}(2, C)$ in Poincarés half-space model of hyperbolic geometry.

In Sec. III we give the spectral resolution of Dirac's equation in an open and simply connected RW cosmology with arbitrary expansion factor. This has been done in Ref. 16 for 
closed RW cosmologies. Evidently, if one replaces in this article the spherical functions by pseudospherical ones, one can get also the spectral resolution for the open models. On the other hand we present the spectral theory in a way that can be easily adapted to multiply connected cosmologies, which do not admit continuous symmetries. We give the spectral resolution in the Poincaré half-space in terms of horospherical elementary waves.

In Sec. IV we give a description of spin in hyperbolic spaces. Spin just appears as a vector field on the wave fronts of the elementary waves. In Sec. V we construct the (anti-) unitary representations of the $C-P-T$ symmetries in $H^{3}$, and discuss their meaning in simply connected RW cosmologies.

The universal covering space of the multiply connected spacelike slices of our RW models is homogeneous, and the semiclassical approximation happens to be exact in it, cf. Sec. VI and Ref. 17 for a more precise statement on that. This phenomenon leads to interesting relations between classical geodesic motion and wave mechanics, cf. Refs. 17 and 18, and in this article we will use it again to clarify the energy concept for spinor fields in (multiply connected) RW cosmologies with negatively curved spacelike slices. In Sec. VI we discuss the meaning of the particle-antiparticle concept and the meaning of the field energy in the context of horospherical waves traveling in the expanding space, and give several examples.

In Sec. VII we discuss spinorial wave fields in multiply connected RW cosmologies, and give explicitly the spectral resolution of the Dirac equation in this context. In Sec. VIII we construct and study space inversions in a multiply connected RW geometry. The discrete symmetries $C, P$, and $T$ still admit spinor representations that are symmetries of the Dirac equation. However, the unitarity of the $P$ operation cannot be retained in the case of a multiply connected space. In Sec. IX we present further discussion, and in the Appendix we summarize our notation and some technical things concerning spinors in hyperbolic spaces.

For the basic concepts of spinor calculus in curved spaces we refer to Refs. 19 and 20. The formalism and the notation concerning covariant spinorial differentiation we have taken over from Ref. 20, which together with Refs. 7 and 17 is a prerequisite, otherwise this article is essentially self-contained. But a certain familiarity with quantum field theory in curved spaces would enhance the understanding of this article, see the standard reviews on this subject, ${ }^{21,22}$ where also an extensive list of more recent references on spinor theories in curved spaces can be found. I add here Ref. 23, where the Gordon decomposition of the current is discussed in the context of spatially flat RW cosmologies. I emphasize however that we will not attempt to do second quantization, nor did we in the preceding articles. ${ }^{7,17,8}$ What we are studying here is wave mechanics, and how it relates to the topology of the underlying space.

\section{TRANSFORMATION THEORY OF THE DIRAC EQUATION IN SIMPLY CONNECTED RW COSMOLOGIES OF NEGATIVE SPATIAL CURVATURE}

We study the Dirac equation on $\mathbf{R}^{(+)} \times H^{3}$, where $\mathbf{R}^{(+)}$denotes the time (semi-)axis, and $H^{3}$ the Minkowski hyperboloid, or equivalently the Poincare half-space. The metric is defined in Eq. (A1); for the basic definitions and our notation we refer to the Appendix and Refs. 7 and 17.

Dirac's equation reads ${ }^{19,20}$

$$
\left(\gamma^{\lambda} \nabla_{\lambda}+\mu\right) \psi=0
$$

with $\gamma^{\mu}$ as in Eq. (A7), $\nabla_{\lambda}$ as in Eq. (A14), and $\mu:=m c / \hbar$.

More explicitly, we may write Eq. (2.1) as

$$
\left[-c^{-2} \tilde{\gamma}_{0} \frac{\partial}{\partial x^{0}}+\frac{x^{3}}{R a(\tau)} \tilde{\gamma}_{i} \frac{\partial}{\partial x^{i}}-\frac{3}{2} \tilde{\gamma}_{0} c^{-2} \frac{\dot{a}}{a}-\frac{1}{R a} \tilde{\gamma}_{3}+\mu\right] \psi=0,
$$


pp. 1576-94 in attached files 


$$
\gamma_{0}:=\tilde{\gamma}_{0}, \quad \gamma_{k}:=\sqrt{g_{33}} \tilde{\gamma}_{k} .
$$

We have again Eq. (A6) with $\tilde{\gamma}^{\mu}$ replaced by $\gamma^{\mu}$, and $\eta_{\mu v}$ by $g_{\mu v}$.

To calculate the connection symbols $\Gamma_{\mu}$ of the spinor connection we follow Ref. 20. At first we find a tetrad $b_{\mu}^{\lambda}(x)$ and its inverse $a_{\mu}^{\lambda}(x)$, so that

$$
\eta_{\lambda \kappa} b_{\mu}^{\lambda} b_{v}^{\kappa}=g_{\mu v}, \quad a_{\kappa}^{\mu} b_{v}^{\kappa}=\delta_{v}^{\mu} .
$$

Then we calculate the connection symbols via the following procedure:

$$
\begin{gathered}
\Gamma_{\kappa}:=\frac{1}{4} B_{\mu v \kappa} s^{\mu v}+A_{\kappa} \text { id, } s^{\mu v}:=\frac{1}{2}\left(\gamma^{\mu} \gamma^{\nu}-\gamma^{v} \gamma^{\mu}\right), \\
B_{\mu v \kappa}:=g_{\mu \lambda}\left(C_{v \kappa}^{\lambda}-\Gamma_{v \kappa}^{\lambda}\right), \quad C_{v \kappa}^{\lambda}:=\frac{\partial b_{v}^{\alpha}}{\partial x^{\kappa}} a_{\alpha}^{\lambda},
\end{gathered}
$$

where $A_{\kappa}(x)$ denotes here an arbitrary vector field, the electromagnetic potential, and id the four by four unit matrix. The $\Gamma_{\mu}$ satisfy

$$
\frac{\partial \gamma_{\mu}}{\partial x^{\nu}}=\Gamma_{\nu \mu}^{\kappa} \gamma_{\kappa}+\Gamma_{v} \gamma_{\mu}-\gamma_{\mu} \Gamma_{v}
$$

and Eq. (A9) is the most general solution of Eq. (A10).

We choose as a solution of Eq. (A8)

$$
b_{0}^{0}=a_{0}^{0}=1, \quad b_{i}^{i}=1 / a_{i}^{i}=\sqrt{g_{33}}
$$

and off-diagonal elements zero. We obtain

$$
\begin{gathered}
\Gamma_{0}=A_{0} \text { id, } \Gamma_{1}=\frac{1}{2}\left(-c^{-2} \frac{R}{t} \dot{a}(\tau) \tilde{\gamma}_{0} \tilde{\gamma}_{1}+\frac{1}{t} \tilde{\gamma}_{1} \tilde{\gamma}_{3}\right)+A_{1} \text { id, } \\
\Gamma_{2}=\frac{1}{2}\left(-c^{-2} \frac{R}{t} \dot{a}(\tau) \tilde{\gamma}_{0} \tilde{\gamma}_{2}+\frac{1}{t} \tilde{\gamma}_{2} \tilde{\gamma}_{3}\right)+A_{2} \text { id, } \quad \Gamma_{3}=-\frac{1}{2} c^{-2} \frac{R}{t} \dot{a}(\tau) \tilde{\gamma}_{0} \tilde{\gamma}_{3}+A_{3} \text { id. }
\end{gathered}
$$

In this article we assume throughout $A_{\kappa}(x)=0$. Finally we note

$$
\begin{gathered}
\gamma^{1} \Gamma_{1}=\gamma^{2} \Gamma_{2}=\frac{1}{2}\left(c^{-2} \frac{\dot{a}}{a} \tilde{\gamma}_{0}+\frac{1}{R a} \tilde{\gamma}_{3}\right), \quad \gamma^{3} \Gamma_{3}=\frac{1}{2} c^{-2} \frac{\dot{a}}{a} \tilde{\gamma}_{0}, \\
\gamma^{\lambda} \Gamma_{\lambda}=\frac{3}{2} c^{-2} \frac{\dot{a}}{a} \tilde{\gamma}_{0}+\frac{1}{R a} \tilde{\gamma}_{3} .
\end{gathered}
$$

The covariant derivatives of a spinor and its adjoint, $\bar{\psi}:=\psi^{\dagger} \gamma^{0}$, are

$$
\nabla_{\lambda} \psi:=\frac{\partial \psi}{\partial x^{\lambda}}-\Gamma_{\lambda} \psi, \quad \nabla_{\lambda} \bar{\psi}:=\frac{\partial \bar{\psi}}{\partial x^{\lambda}}+\bar{\psi} \Gamma_{\lambda} ;
$$

an exposition of spinorial covariant differentiation can be found in Ref. 20, see also Ref. 21 and the literature cited therein.

\footnotetext{
${ }^{1}$ P. Jordan, Schwerkraft und Weltall, 2nd ed. (Vieweg, Braunschweig, 1955), p. 113.

${ }^{2}$ E. Schrödinger, Physica 6, 899 (1939).

${ }^{3}$ J. A. Wheeler, in The Physicist's Conception of Nature, edited by J. Mehra (Reidel, Dordrecht, 1973).
} 
${ }^{4}$ F. J. Dyson, Rev. Mod. Phys. 51, 447 (1979).

${ }^{5}$ S. Weinberg, Gravitation and Cosmology (Wiley, New York, 1972).

${ }^{6}$ C. W. Misner, K. S. Thorne, and J. A. Wheeler, Gravitation (Freeman, New York, 1973).

${ }^{7}$ R. Tomaschitz, J. Math. Phys. 32, 2571 (1991).

${ }^{8}$ R. Tomaschitz, J. Math. Phys. 34, 3133 (1993).

${ }^{9}$ R. Tomaschitz in Deterministic Chaos in General Relativity, edited by D. Hobill (Plenum, New York, 1994).

${ }^{10}$ W. Thurston, Bull. Am. Math. Soc. 6, 357 (1982).

${ }^{11} \mathbf{R}$. Tomaschitz, in Group Theoretical Methods in Physics, Proceedings of the XIX International Colloquium, edited by M. A. del Olmo et al. (CIEMAT, Madrid, 1993).

${ }^{12}$ L. Parker, Phys. Rev. D 5, 2905 (1972).

${ }^{13}$ E. M. Corson, Introduction to Tensors, Spinors, and Relativistic Wave Equations (Blackie \& Son, London, 1953).

${ }^{14}$ J. D. Bjorken and S. D. Drell, Relativistic Quantum Mechanics (McGraw-Hill, New York, 1964).

${ }^{15}$ R. Tomaschitz, Complex Syst. 6, 137 (1992).

${ }^{16}$ E. Schrödinger, Eigenschwingungen des Sphärischen Raumes, Collected Papers, Vol. II (Vieweg, Wiesbaden, 1984), pp. 227-270.

${ }^{17}$ R. Tomaschitz, J. Math. Phys. 34, 1022 (1993).

${ }^{18}$ R. Tomaschitz, Int. J. Theor. Phys. 33, 355 (1994).

${ }^{19}$ E. Schrödinger, Sitzungsber. Preuss. Akad. Wiss. Berlin 11-12, 105 (1932).

${ }^{20}$ V. Bargmann, Sitzungsber. Preuss. Akad. Wiss. Berlin 11-12, 346 (1932).

${ }^{21}$ N. D. Birrell and D. C. W. Davies, Quantum Fields in Curved Space (Cambridge University, Cambridge, England, 1982).

${ }^{22}$ G. W. Gibbons, in General Relativity: An Einstein Centenary Survey, edited by S. W. Hawking and W. Israel (Cambridge University, Cambridge, England, 1979).

${ }^{23}$ A. O. Barut and I. H. Duru, Phys. Rev. D 36, 3705 (1987).

${ }^{24}$ A. F. Beardon, The Geometry of Discrete Groups (Springer, New York, 1983).

${ }^{25}$ B. Maskit, Kleinian Groups (Springer, New York, 1986).

${ }^{26}$ L. D. Landau and E. M. Lifshitz, The Classical Theory of Fields (Pergamon, Oxford, 1962), Sec. 100.

${ }^{27}$ N. E. Hurt, Geometric Quantization in Action (Reidel, Dordrecht, 1983).

${ }^{28}$ H. Petersson, Math. Ann. 115, 23 (1938).

${ }^{29}$ H. Maass, Math. Ann. 121, 141 (1949).

${ }^{30}$ D. A. Hejhal, The Selberg Trace Formula for PSL(2,R) (Springer, New York, 1983), Vol. II (LN, Vol. 1001).

${ }^{31} \mathrm{~J}$. Lehner, Discontinuous Groups and Automorphic Functions (American Mathematical Society, Providence, RI, 1964).

${ }^{32}$ S. J. Patterson, Compositio Math. 31, 83 (1975).

${ }^{33}$ S. J. Patterson, Arkiv Mat. 226, 167 (1988).

${ }^{34}$ R. Tomaschitz, Physica D 34, 42 (1989).

${ }^{35}$ J. Stillwell, Classical Topology and Combinatorial Group Theory (Springer, New York, 1980).

${ }^{36}$ R. Tomaschitz, Int. J. Theor. Phys. 31, 187 (1992).

${ }^{37}$ R. Fricke and F. Klein, Vorlesungen über die Theorie der Automorphen Funktionen (Teubner, Leipzig 1897, also Johnson Reprint, New York, 1965), Vol. I, pp. 200-209.

${ }^{38}$ L. Bers, in Proceedings of the International Congress of Mathematicians, 1958, edited by J. A. Todd (Cambridge University, Cambridge, England, 1960).

${ }^{39}$ D. A. Hejhal, The Selberg Trace Formula for PSL(2,R) (Springer, New York, 1976), Vol. I (LN, Vol. 548).

${ }^{40}$ G. C. McVittie, General Relativity and Cosmology (Chapman and Hall, London, 1965), pp. 170-71. 